\title{
HCC-ART score, a simple, highly sensitive and specific test for early diagnosis of hepatocellular carcinoma: a large-scale, multicentre study
}

\author{
A M Attallah ${ }^{*}, 1$ M M Omran ${ }^{2}$, A A Attallah ${ }^{1}$, S O Abdallah ${ }^{3}$, K Farid $^{4}$, H Darwish ${ }^{5}$, I El-Dosoky ${ }^{6}$ \\ and Y M Shaker ${ }^{7}$ \\ ${ }^{1} R$ \& D Department, Biotechnology Research Center, PO Box (14), 23 July Street, Industrial Zone, New Damietta 34517, Egypt; \\ ${ }^{2}$ Faculty of Science, Helwan University, Cairo, Egypt; ${ }^{3}$ Faculty of Science, Cairo University, Giza, Egypt; ${ }^{4}$ Tropical Medicine \\ Department, Faculty of Medicine, Mansoura University, Mansoura, Egypt; ${ }^{5}$ Damietta Oncology Center, Damietta, Egypt; \\ ${ }^{6}$ Pathology Department, Faculty of Medicine, Mansoura University, Mansoura, Egypt and ${ }^{7}$ National Research Center, Cairo, Egypt
}

Background: A simple scoring system is needed to discriminate HCC from patients with chronic liver diseases (CLD). The simplest score would be one that requires only variables that can be documented simply from routine laboratory tests without the need for sophisticated tests.

Methods: Data from the estimation group (1351 patients) and the validation group (2208 patients) were retrospectively analysed. Liver fibrosis-negative control and liver cirrhosis were compared with HCC. Area under ROC curve (AUC) were used to develop HCC- $\alpha$-fetoprotein-routine test (HCC-ART).

Results: Hepatocellular carcinoma-AFP-routine test showed diagnostic accuracy for liver cirrhosis vs HCC with ROC curves of $0.99 \%$, sensitivity of $97 \%$, and specificity of $96 \%$ in the estimation, and $0.95 \%, 90 \%$, and $83 \%$, respectively, in the validation. Sensitivity (97\%) and specificity (100\%) were obtained to discriminate HCC from liver fibrosis. Area under curve for AFP at $400 \mathrm{UI}-1$ was 0.70 , sensitivity was $41 \%$, and specificity was $99 \%$ in the estimation, and $0.77 \%, 54 \%$, and $99 \%$, respectively, in the validation. The AUC for HCC-ART in HCC with single tumour, absent vascular invasion, size $<2 \mathrm{~cm}$ and CLIP score (0-1) were 0.95, 0.93, 0.86, 0.87 , respectively, compared with $0.72,0.71,0.71,0.50$, respectively, for AFP.

Conclusion: Hepatocellular carcinoma-AFP-routine test could increase the accuracy of HCC screening and surveillances and could be used worldwide without extra efforts.

The current diagnosis of HCC is a multistage process that includes clinical, laboratory, imaging and pathological examinations (Gonzalez and Keeffe, 2011). The use of AFP as a screening test for early HCC is doubtful on account of its sensitivity of 39-64\%, specificity of $76-91 \%$, and low positive predictive value of $9-32 \%$ (Lai et al, 2012). Des- $\gamma$-carboxy prothrombin (DCP) as well as lens culinaris agglutinin-reactive fraction of AFP (AFP-L3), MAGE-4 protein, and $\alpha$-fucosidase have been developed with the intent to detect HCC (Kew, 2012). Imaging has a key role in the diagnosis of HCC (Manichon et al, 2012). The imaging findings are strongly influenced by the methods used, the skill of the examiner, and the quality of the imaging apparatus (Murakami et al, 2001; Choi and Lee, 2010). The sensitivity and positive predictive value can be as low as $35 \%$ and $15 \%$, respectively, in some cases with cirrhosis 
(Barreiros et al, 2012). Identification of novel serum biomarkers is an important goal in the diagnosis of cancer, especially for detection and screening in early-stage cancer. The aim of this study was to develop and validate the diagnostic accuracy of HCC-ART score based on age, AFP, aspartate aminotransferase (AST)/alanine aminotransferase (ALT) ratio (AAR), alkaline phosphatase (ALP), and albumin (Alb) for HCC detection in comparison with AFP.

\section{MATERIALS AND METHODS}

Patients were divided into two groups based on the time of assessment: the estimation group $(n=1351)$ and the validation group $(n=2208)$. The estimation population consisted of patients from the Tropical Medicine Unit (Mansoura University Hospitals, Mansoura, Egypt). In this retrospective study, all patients with chronic hepatitis $\mathrm{C}$ were tested positive for the presence of anti$\mathrm{HCV}$ antibodies using ETI-AB-HCVK-3 kit (Sorine Biomedica, Saluggia, Italy) and HCV-RNA using quantitative polymerase chain reaction assay (COBAS Ampliprep/COBAS TaqMan; Roche Diagnostics, Pleasanton, CA, USA). Subjects were divided into two main groups: group I - the HCC group included 227 cirrhotic patients with proved HCC (110 males and 117 females). The diagnosis of HCC in those patients was carried out according to the American Association for the Study of Liver Diseases (AASLD) Practice Guidelines (Bruix and Sherman, 2005). The diagnosis of HCC was based on AFP levels $\geqslant 400 \mathrm{Ul}^{-1}$, presence of hepatic focal lesion (s) detected by liver ultrasound (US), and confirmed by computed tomography (CT) and/or magnetic resonance imaging (MRI) techniques. The final diagnosis was confirmed by histopathologic analysis on US-assisted fine-needle biopsy, when indicated. Hepatocellular carcinoma staging was conducted as follows: CLIP score, based on four items and with a score ranging from 0 to 6 . These four items included: (I) Child-Pugh stage $(\mathrm{A}=0, \mathrm{~B}=1$, and $\mathrm{C}=2)$ (Pugh et al, 1973); (II) tumour morphology (uninodular $<50 \%, 0$; multinodular $<50 \%$, 1 ; and massive or $>50 \%, 2)$; (III) AFP level $\left(<400 \mathrm{UL}^{-1}, 0\right.$; $\left.>400 \mathrm{UL}^{-1}, 1\right)$; and (IV) presence of portal vein thrombosis (no, 0; yes, 1) (CLIP, 2000). Group II - The non-malignant chronic liver disease (CLD) group included 1124 patients with chronic hepatitis (836 males, 288 females). Diagnosis of CLD in this group was based on the standard clinical, biochemical, and ultrasonographic criteria, as well as the pathological data. Metavir scoring system was used to stage fibrosis (F0-F4) (Poynard et al, 1997). Fibrosis was scored on a five-point scale: F0, no fibrosis; F1, portal fibrosis alone; F2, portal fibrosis with rare septae; F3, portal fibrosis with many septae; and F4, cirrhosis. A questionnaire of several items was completed for every patient and histological data was obtained at liver biopsy. Patients with the following conditions were excluded from the study: presence of other causes of liver diseases, hepatitis B virus (HBV) infection, or other suspected malignancies. None of the HCC patients had received transarterial embolisation or chemotherapy or underwent radiofrequency ablation or surgical interference.

Validation study. The clinical and pathological classification adopted for validation study was the same as those used in patients in the estimation study. All patients and controls signed an informed written consent after having explained about the aim and details of the study. The HCC-ART score was applied to a validation group comprising 2208 patients to confirm the accuracy and reproducibility in a subsequent different, but related group of patients. Patients included in the validation study were divided into two main groups: group I - the HCC group included 537 patients (417 males, 120 females) from Damietta Oncology Center, Damietta, Egypt; group II - the non-malignant CLD group included 1671 patients with chronic hepatitis (1273 men, 398 females) from Tropical Medicine Unit, Faculty of Medicine, Mansoura University, Mansoura, Egypt. It also included more F4 patients, such as child A (83 patients) and child B (25 patients). The study protocol was approved by the Institutional Review Committee and conformed to the ethical guidelines of the 1975 Helsinki Declaration.

Laboratory tests. Fasting blood samples were collected from all patients and divided into three parts; one part was collected without an anticoagulant. Sera were separated and tested fresh for liver function tests. Liver function tests such as Alb, AAR, and ALP were measured on an automated biochemistry analyzer (Hitachi 917; Roche Diagnostics). $\alpha$-Fetoprotein level was performed by chemiluminescence, with Immulite AFP (1000) kit (Diagnostic Products Corporation, Los Angeles, CA, USA). In addition, serologic tests were carried out for HCV using commercial ELISA kits (ETI-AB-HCVK-3 kit; Sorine Biomedica, Suluggia, Italy). The second part of blood was drawn into KEDTA tubes for complete blood count. Complete blood count was performed on KX-21 Sysmex automated hematology analyzer (Sysmes Corporation, Kobe, Japan). The third portion was treated with a citrate solution for prothrombin-INR (international normalised ratio).

Statistical analysis. All statistical analyses were carried out by a Statistical Package for the Social Sciences (SPSS), version 15.0 on Microsoft Windows XP (SPSS Inc., Chicago, IL,USA). Continuous variables were expressed as mean \pm s.d., whereas categorical variables were expressed as numbers (percentages). A value of $P<0.05$ was considered statistically significant. The correlation was evaluated by Pearson's correlation coefficient. All analytes had a gaussian distribution in patients with liver diseases; the deviation of AFP was successfully corrected by log transformation of the data. Patients were divided into several groups. The main end point was the identification of patients with HCC vs liver cirrhosis (F4). In secondary analyses, patients were also classified into liver fibrosis (F1-F3) and no liver fibrosis (F0). First, factors that differed significantly between these groups were identified by univariate analyses: $\chi^{2}$, analysis of variance, Student's $t$-test, or Mann-Whitney $U$-test. The independent discriminative value of blood markers for the diagnosis of HCC was then assessed by ROC curves and stepwise multivariate discriminant analysis. The third step was to construct a score that combined the independent factors. The best score for discrimination was the logistic regression function that combined the most discriminatory independent factors. The best cutoff values for optimal prediction of HCC were determined from the ROC curves. To develop a simplified score, we have found different derived simplified scores for the assessment of liver fibrosis stages without coefficients such as King's score (Cross et al, 2009), FIB-4 (Vallet-Pichard et al, 2007), FibroQ (Hsieh et al, 2009), and FCI score (Ahmad et al, 2011). In our study, markers used in the original HCC-ART were combined to derive simplified score to predict HCC. So this simplified score is different from the HCC-ART original score with coefficients. In the simplified HCC-ART score, we put the positive correlation parameters (age, log AFP, AST/ALT ratio, and ALP) in the numerator and negative correlation parameters (Alb) in the denominator to formulate a simplified score. Common indicators of the candidate blood markers and the model performance (sensitivity, specificity, efficiency, and positive and negative predictive values) were derived from a $2 \times 2$ contingency table.

\section{RESULTS}

Patient characteristics. The clinical background of HCC and nonmalignant CLD patients in the estimation group are shown in Table 1. Hepatocellular carcinoma patients were older than the CLD patients. Hepatocellular carcinoma patients produced a range 


\begin{tabular}{|c|c|c|c|c|c|}
\hline \multicolumn{4}{|c|}{ Non-malignant chronic liver diseases $(n=1124)$} & \multirow[b]{2}{*}{$\operatorname{HCC}(\mathbf{N}=227)$} & \multirow[b]{2}{*}{$\boldsymbol{P}$-value ${ }^{b}$} \\
\hline Variables $^{a}$ & $\mathrm{FO}^{\mathrm{c}}(\mathbf{N}=107)$ & $F 1-F 3^{c}(N=676)$ & $\mathrm{F} 4^{\mathrm{c}}(\mathbf{N}=341)$ & & \\
\hline Age (years) & $37.2 \pm 8.7$ & $42.7 \pm 7.9$ & $46.1 \pm 7.6$ & $59 \pm 10.3$ & $<0.0001$ \\
\hline $\operatorname{AST}\left(U I^{-1}\right)$ & $34.6 \pm 14.2$ & $41.9 \pm 26.6$ & $71 \pm 43$ & $128 \pm 109$ & $<0.0001$ \\
\hline $\operatorname{ALT}\left(\mathrm{UI}^{-1}\right)$ & $40.2 \pm 23.1$ & $49.6 \pm 34.7$ & $77.6 \pm 53.6$ & $67.6 \pm 61.0$ & 0.31 \\
\hline AAR & $0.85 \pm 0.44$ & $0.97 \pm 0.47$ & $1.1 \pm 0.45$ & $1.9 \pm 1.53$ & $<0.0001$ \\
\hline $\operatorname{ALP}\left(U I^{-1}\right)$ & $87.1 \pm 47$ & $83.8 \pm 47.6$ & $94 \pm 40$ & $260 \pm 227$ & $<0.0001$ \\
\hline Albumin $\left(\mathrm{gl}^{-1}\right)$ & $43.9 \pm 3.5$ & $42.7 \pm 3.7$ & $38 \pm 4.2$ & $29 \pm 5.9$ & $<0.0001$ \\
\hline Total bilirubin $\left(\mathrm{mg} \mathrm{dl}^{-1}\right)$ & $0.84 \pm 0.38$ & $0.83 \pm 0.33$ & $1.11 \pm 0.68$ & $4.1 \pm 2.9$ & $<0.0001$ \\
\hline Platelet count $\left(\times\left. 10^{9}\right|^{-1}\right)$ & $238 \pm 56$ & $195 \pm 54$ & $155 \pm 53$ & $135 \pm 92$ & 0.75 \\
\hline Prothrombin-INR & $1.10 \pm 0.12$ & $1.20 \pm 0.20$ & $1.23 \pm 0.15$ & $1.30 \pm 0.20$ & 0.06 \\
\hline$\alpha$-Fetoprotein $\left(\mathrm{UI}^{-1}\right)$ & $2.3 \pm 1.1$ & $0.58 \pm 0.44$ & $38.8 \pm 21.2$ & $32341 \pm 10109$ & $<0.0001$ \\
\hline $\log \alpha$-fetoprotein $\left(U I^{-1}\right)$ & $0.31 \pm 0.23$ & $0.49 \pm 0.3$ & $1.1 \pm 0.44$ & $2.7 \pm 1.38$ & $<0.0001$ \\
\hline \multicolumn{6}{|c|}{ 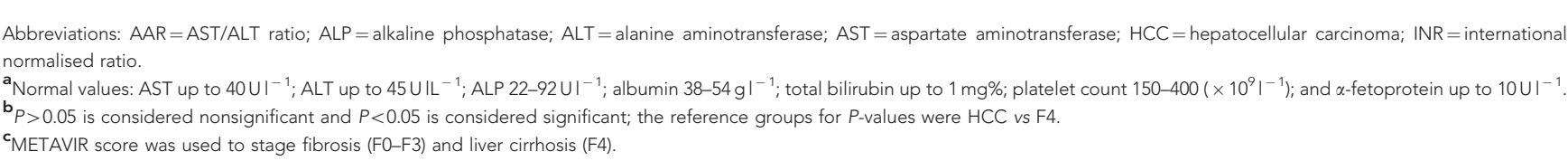 } \\
\hline
\end{tabular}

of AFP values from normal to more than $35268 \mathrm{Ul}^{-1}$. Normal AFP levels are present in as many as $13 \%$ of patients at time of diagnosis and usually remain low, even with advanced HCC. $\alpha$-fetoprotein $>400 \mathrm{Ul}^{-1}$ is considered diagnostic for HCC; $41 \%$ of HCC patients may generate levels that are high. With values of that magnitude, the specificity of AFP is close to $99 \%$. The main end point was the identification of patients with HCC vs patients with liver cirrhosis. Univariate analysis of all variables tested in the estimation study revealed that ALT, platelet count, and prothrombin-INR were nonsignificantly $(P>0.05)$ between HCC and liver cirrhosis patients.

Diagnostic performance of AFP and candidate markers. The AUC was calculated for providing the accuracy of the routine blood markers for differentiating HCC from liver cirrhosis (Figure 1A). The AUC of candidate HCC markers is in the order of Alb (0.85) > ALP (0.76) > age (0.75) > AAR (0.73) > AFP (0.72). Univariate logistic regression analysis of the variables tested in the estimation group set revealed that AFP, AAR, age, ALP, and Alb were associated with HCC. The total bilirubin was then excluded from subsequent analysis. Receiver operating characteristic curves showed that the optimum diagnostic cutoff for AAR, AFP, ALP, age, and Alb were 1.2, 400, 140, 50, and 35, respectively, with odds ratio $(95 \% \mathrm{CI})$ of $5.3,115,11.6,7.4$, and 79 , respectively.

Predictive model. We developed a novel index for discriminating patients with HCC named HCC-ART. It can be represented as: HCC-ART score $=(2.17+((\log$ AFP -1$) \times 10 \times 0.117)+\mathrm{AST} /$ ALT ratio $\times 0.025+$ age $\left.\times 0.012+\mathrm{ALP}\left(\mathrm{Ul}^{-1}\right) \times 0.001\right)-(\mathrm{Alb}$ $\left.\left(\mathrm{gl}^{-1}\right) \times 0.015\right)$. There is no correlation between measured AFP levels and other candidate markers used in the HCC-ART score. They are not related, which means that there is no redundancy and that they explore different biochemical abnormalities associated with the two conditions. There is correlation between measured HCC-ART score and blood markers used in the score (Figure 1B). The relationship between liver diseases (F0, F1-F3, F4, and HCC) and HCC-ART score is illustrated in (Figure 1C). The mean \pm s.d. of HCC-ART score in no liver fibrosis (F0), liver fibrosis (F1-F3) liver cirrhosis (F4), and HCC were $1.95 \pm 0.13,2.1 \pm 0.13$, $2.25 \pm 0.19,3.02 \pm 0.38$, respectively. The HCC-ART can be used equally effectively in both men and women without significant difference $(P>0.05)$. This model provides a high AUC $(0.99 \%)$ for the prediction of HCC (Figure 1D). Similarly, the area under the ROC of HCC and other negative controls (liver fibrosis (F1-F3)) and no liver fibrosis (F0) were 0.99 and 1.0 for HCC-ART (Figure $1 \mathrm{E}$ and $\mathrm{F}$ ). On the basis of the ROC analysis, the optimal cutoff point for HCC-ART to differentiate HCC from non-malignant CLD was 2.5 with odds ratio of 810 . At this cutoff, the sensitivity, specificity and accuracy for differentiating HCC from nonmalignant liver cirrhosis were 97\%, 96\%, and 96\%, respectively (Table 2). Absolute specificity (100\%) was obtained to discriminate HCC from liver fibrosis (F1-F3) and no liver fibrosis (F0). Table 2 presents the calculated sensitivities, specificities, diagnostic accuracy, and positive and negative predictive values for the HCC-ART compared with AFP at $400 \mathrm{Ul}^{-1}$. The values were based on the differentiation between cirrhosis and HCC. The detection using an HCC-ART produced better sensitivity (97\%) and specificity (96\%) compared with $41 \%$ and $99 \%$ for AFP at $>400 \mathrm{Ul}^{-1}$. Absolute specificity (100\%) was obtained to discriminate HCC from liver fibrosis (F1-F3) and no liver fibrosis (F0) (Table 2).

Diagnostic performances of HCC-ART score without coefficient. To simplify HCC-ART, we devised a novel index, called simplified HCC-ART without numerical constant and coefficients: simplified HCC-ART $=\left(\right.$ age $($ years $) \times \log$ AFP $\left(\mathrm{Ul}^{-1}\right)$ $\left.\times \operatorname{AAR} \times \operatorname{ALP}\left(\mathrm{Ul}^{-1}\right)\right) /\left(\mathrm{Alb}\left(\mathrm{gl}^{-1}\right)\right)$. Table 2 presents the calculated sensitivities, specificities, diagnostic accuracy, and positive and negative predictive values for the simplified HCCART at a selected cutoff score $=280$ (i.e. $<280$ indicated absence of HCC and $>280$ indicated presence of HCC). Receiver operating characteristic curves of simplified HCC-ART showed AUC of 0.95, sensitivity of $92 \%$, and specificity of $91 \%$ in the estimation group.

Validation study. The HCC-ART was further assessed for their diagnostic power and discriminative ability in an independent and larger validation set. The clinical background of HCC and nonmalignant CLD patients in the validation group is shown in Table 3. The majority of liver cirrhosis patients were classified as compensated liver function Child-Pugh Class A, 420 (94\%) and Child-Pugh Class B, 25 (6\%). The majority of HCC patients were 
A

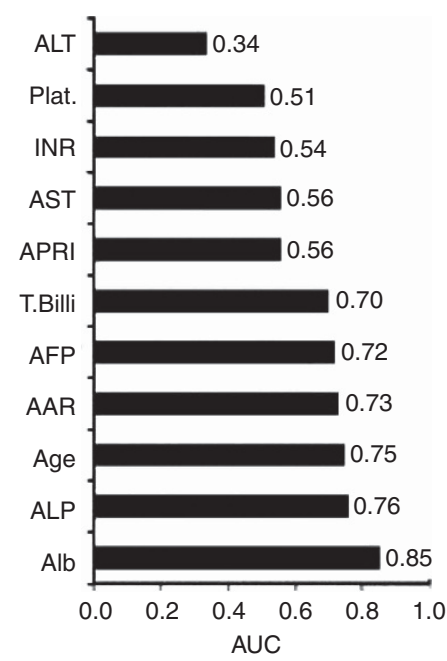

D

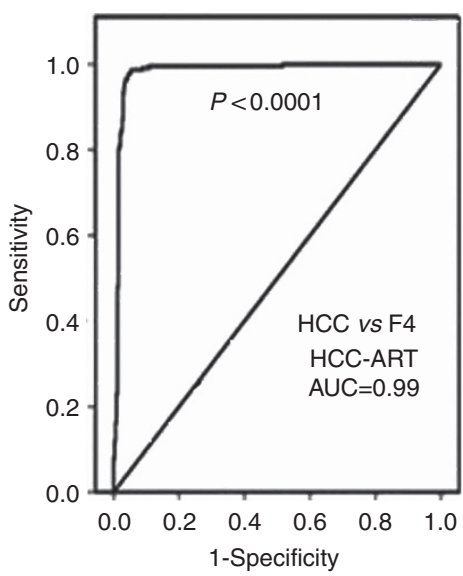

B

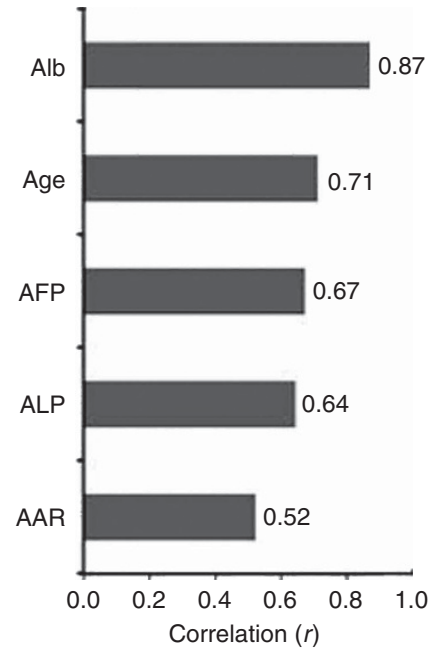

E

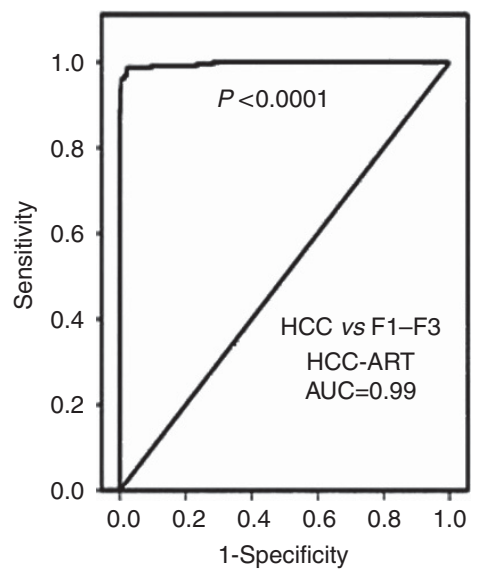

C

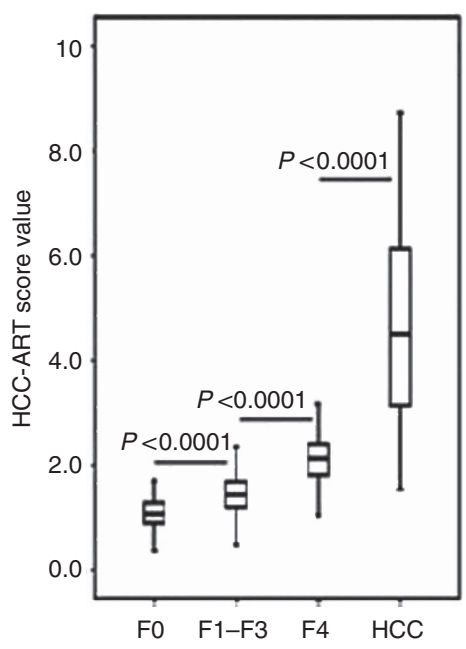

$\mathbf{F}$

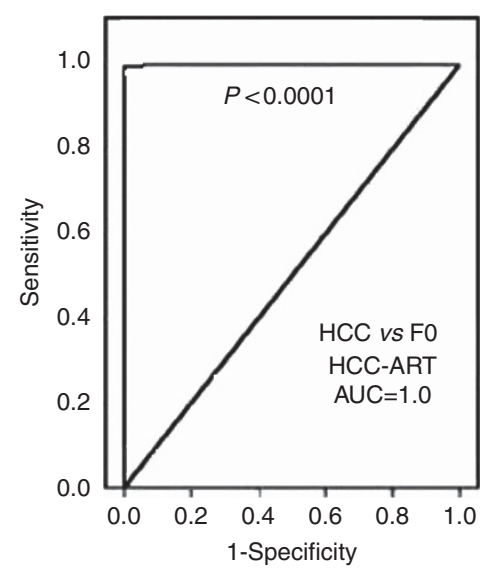

Figure 1. Development of HCC-ART score in 1351 patients with non-malignant CLDs and HCC in the estimation group. (A) Area under curve of candidate's markers to discriminate patients with HCC from patients with liver cirrhosis. (B) Development of HCC-ART score combining Alb, age, AFP, ALP, and AST/ALT ratio, correlation between the different markers included in the score, and the final HCC-ART score. (C) Box plots of HCC-ART score to discriminate HCC from no fibrosis (F0), liver fibrosis (F1-F3), and liver cirrhosis (F4) patients. The whiskers indicate the highest and lowest values, and the line across the box indicates the median value. (D) The AUC of HCC-ART score to discriminate 227 patients with HCC from 341 patients with liver cirrhosis (F4). (E) The AUC of HCC-ART score to discriminate 227 patients with HCC from 676 patients with liver fibrosis (F1-F3). (F) The AUC of HCC-ART score to discriminate 227 patients with HCC from 107 patients with no liver fibrosis (F0). Abbreviations: $A A R=A S T-A L T$ ratio; $A F P=\alpha$-fetoprotein; $A P R I=A S T /$ platelets ratio index; Plat. $=$ platelet count; $T$. bili $=$ total bilirubin .

classified as minimal or intermediate according to the CLIP staging. Overall, 128 out of 537 (24\%) patients had CLIP 1, 255 of 537 (47\%) had CLIP 2, 122 of 537 (23\%) had CLIP 3, 29 of 537 (5\%) had CLIP 4, and 3 of 537 (1\%) had CLIP 5. The diagnostic value of the HCC-ART was still high, with an area under the ROC curve for discriminating patients with HCC from those with liver cirrhosis was 0.95; for discriminating patients with HCC from patients with F1-F3 was 1.0; and for discriminating patients with HCC from patients with F0 was 1.0 (Figure $2 \mathrm{~B}$ and Table 4). The diagnostic power of the HCC-ART in tumour burden features, vascular invasion, size of nodules, and CLIP score across the validation group was reported in Table 4 . The values were based on the differentiation between cirrhosis (child A and child B) and tumour burden of HCC. The AUC for HCC-ART in patients with HCC who had only single tumour, absent vascular invasion, size $2 \mathrm{~cm}$ or less, and CLIP $(0-1)$ were $0.95,0.93,0.86,0.87$, respectively, compared with $0.72,0.71,0.71,0.50$, respectively, for AFP (Figure 2). Table 5 presents the calculated sensitivities, specificities, and diagnostic accuracy for AFP (at cutoff $400 \mathrm{Ul}^{-1}$ ).
The diagnostic power of AFP alone in tumour burden features, vascular invasion, size of nodules, and CLIP score across the validation group appeared inferior to that of the HCC-ART. We used CLIP score (0-1) to define early stages of HCC. To stage patients in the CLIP score, multiple clinical indexes, such as ChildPugh score, tumour morphology, AFP level and presence of portal vein thrombosis, are taken into account. The AFP sensitivity was 1 for CLIP 0-1 and can be explained as follows: CLIP score used a high constant value of AFP $\left(400 \mathrm{Ul}^{-1}\right)$, which is not common with early (CLIP 0-1) cases of HCC. The value of AFP in 127 HCC cases with child $\mathrm{B}$ and had CLIP score $0-1$ was $<400 \mathrm{Ul}^{-1}$, and then AFP was not diagnostic for these cases. The value of AFP was more than $400 \mathrm{Ul}^{-1}$ in only one case (child A). Then, the final sensitivity was $<1 \%$ (Table 5 ). The mean \pm s.d. of AFP for the total 128 cases was $25.4 \pm 49.5 \mathrm{Ul}^{-1}$. Table 6 shows the results of the simplified score. Receiver operating characteristic curves of simplified HCC-ART showed AUC of 0.91 , sensitivity of $83 \%$, and specificity of $86 \%$ in the validation group. When simplified HCC-ART was applied to HCC patients with only single tumour, 
Table 2. Diagnostic performances of AFP and HCC-ART score to discriminate 227 patients with HCC from patients with non-malignant chronic liver diseases $(n=1124)$

\begin{tabular}{|c|c|c|c|c|c|c|}
\hline & Sen (\%) & Spe (\%) & PPV (\%) & $\operatorname{NPV}(\%)^{a}$ & $A C(\%)$ & AUC \\
\hline \multicolumn{7}{|l|}{$\mathrm{AFP} \geqslant 400 \mathrm{UI}^{-1}$} \\
\hline $\begin{array}{l}\text { HCC vs } 341 \text { liver cirrhosis (F4) } \\
\text { HCC vs } 676 \text { liver fibrosis (F1-F3) } \\
\text { HCC vs } 107 \text { no liver fibrosis (F0) }\end{array}$ & 41 & $\begin{array}{r}99 \\
100 \\
100\end{array}$ & $\begin{array}{r}98 \\
100 \\
100\end{array}$ & $\begin{array}{l}74 \\
83 \\
48\end{array}$ & $\begin{array}{l}78 \\
84 \\
61\end{array}$ & $\begin{array}{l}0.70 \\
0.71 \\
0.70\end{array}$ \\
\hline \multicolumn{7}{|l|}{ HCC-ART score at cutoff $\geqslant 2.5^{b}$} \\
\hline $\begin{array}{l}\text { HCC vs } 341 \text { liver cirrhosis (F4) } \\
\text { HCC vs } 676 \text { liver fibrosis (F1-F3) } \\
\text { HCC vs } 107 \text { no liver fibrosis (FO) }\end{array}$ & 97 & $\begin{array}{r}96 \\
100 \\
100\end{array}$ & $\begin{array}{r}94 \\
100 \\
100\end{array}$ & $\begin{array}{l}98 \\
99 \\
95\end{array}$ & $\begin{array}{l}96 \\
98 \\
99\end{array}$ & $\begin{array}{l}0.99 \\
0.99 \\
1.0\end{array}$ \\
\hline \multicolumn{7}{|c|}{ Simplified HCC-ART score at cutoff $\geqslant 280^{c}$} \\
\hline $\begin{array}{l}\text { HCC vs } 341 \text { liver cirrhosis (F4) } \\
\text { HCC vs } 676 \text { liver fibrosis (F1-F3) } \\
\text { HCC vs } 107 \text { no liver fibrosis (F0) }\end{array}$ & 92 & $\begin{array}{r}91 \\
100 \\
100\end{array}$ & $\begin{array}{r}87 \\
99 \\
100\end{array}$ & $\begin{array}{l}95 \\
97 \\
86\end{array}$ & $\begin{array}{l}92 \\
98 \\
95\end{array}$ & $\begin{array}{l}0.95 \\
0.98 \\
0.98\end{array}$ \\
\hline
\end{tabular}

Table 3. Clinical data of the validation study $(n=2208)$

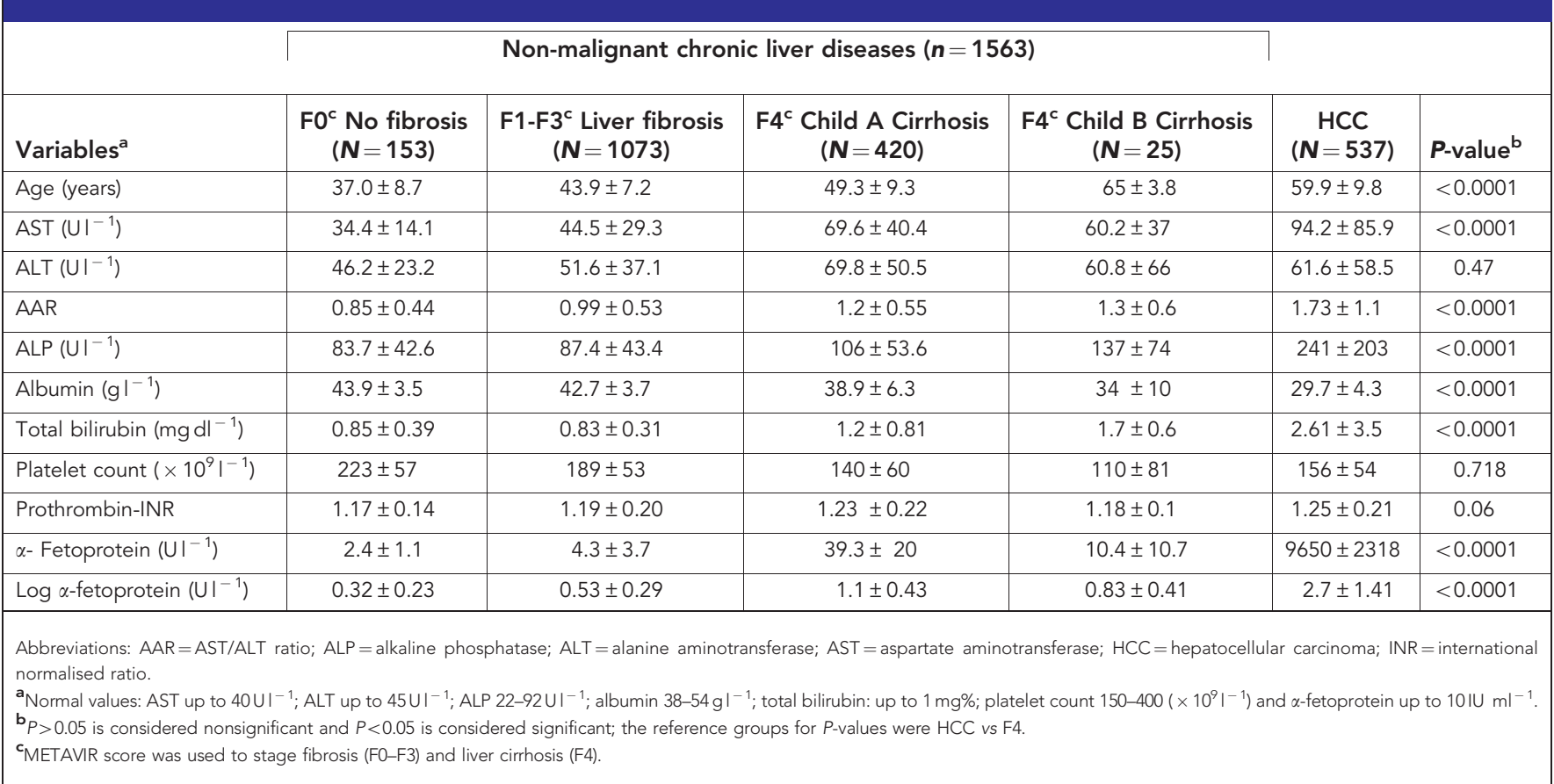

absent vascular invasion, size $2 \mathrm{~cm}$ or less and CLIP $(0-1)$, the results were reproduced without significant differences $(P>0.05)$.

\section{DISCUSSION}

Hepatocellular carcinoma is strongly linked to hepatitis B virus and hepatitis $\mathrm{C}$ virus. In Egypt, prevalence's for HBV and HCV infection were $6.7 \%$ and $13.9 \%$, respectively, among healthy populations, and $25.9 \%$ and $78.5 \%$, respectively, among HCC cases. Adults had higher prevalence's of both infections (adult $\mathrm{HBV}=8.0 \%$, child $\mathrm{HBV}=1.6 \%$; adult $\mathrm{HCV}=15.7 \%$, child $\mathrm{HCV}=4.0 \%$ ) (Lehman and Wilson, 2009). We based our study in clinical centres in Egypt where most cases of HCC are related to $\mathrm{HCV}$ infection. In this retrospective study, all patients with HCC were positive for anti-HCV. Hepatocellular carcinoma developed on top of HCV-related liver cirrhosis. This is in agreement with many authors (Lehman and Wilson, 2009). Unlike other solid malignancies, the prognosis of HCC is not solely dependent on 


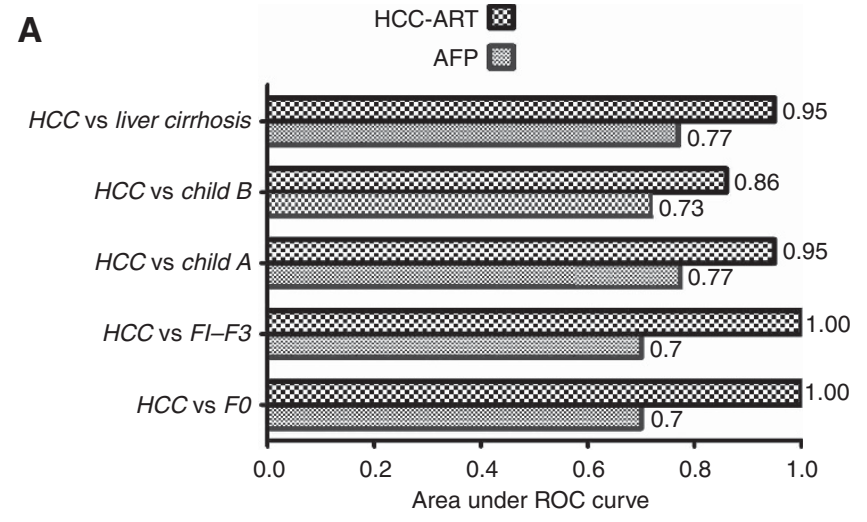

B

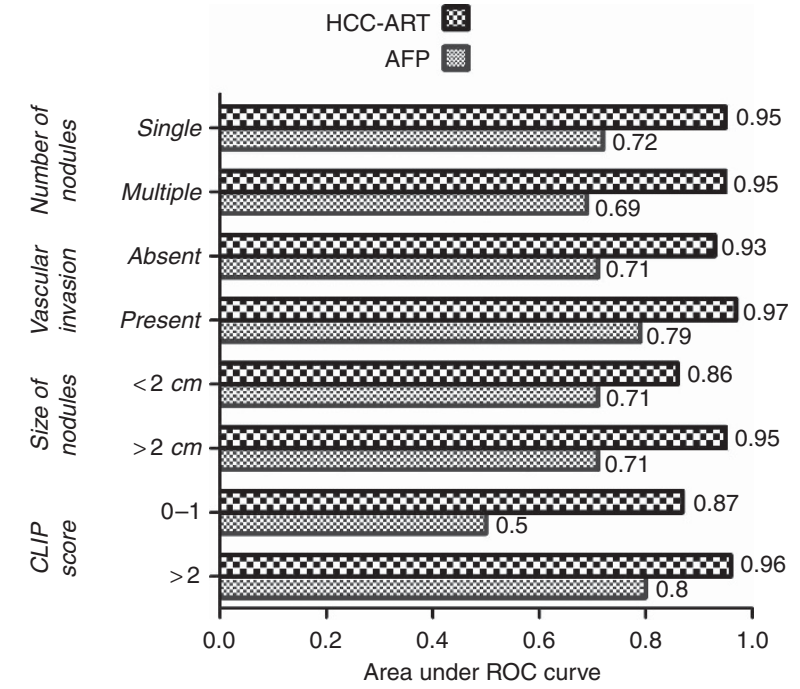

Figure 2. Area under ROC curve (AUC) of validated HCC-ART score compared with AFP $\geqslant 400 / /^{-1}$. (A) To discriminate 537 patients with HCC from patients with non-malignant CLDs $(n=1671)$.

(B) Diagnosis of early HCC with tumour burden features such as number of nodules, vascular invasion, size of nodules, and CLIP score.

tumour burden but is also adversely influenced by impaired liver function secondary to the underlying pathogenic condition (Tandon and Garcia-Tsao, 2009). Several staging systems have been proposed to predict patient prognosis, including parameters such as functional liver reserve, performance status, circulating tumour markers, and extent of spread of the primary tumour (Bruix et al, 2001). Several models to guide prognosis in HCC have been developed, each one including parameters reflecting both liver dysfunction and tumour stage. None of these previous prognostic indices are considered ideal, and despite the increasing number of published comparative studies, there is no consensus on the optimal system that should be utilised (Camma and Cabibbo, 2009). Our study was undertaken to develop a clinically useful HCC-ART score for the diagnosis of patients with HCC. To maximise the clinical use of this score, HCC-ART was designed to include simple, non-invasive, and routinely measured markers. There are no reports examining the diagnostic value of these markers (age, AFP, AAR, ALP, and Alb) combined in a score for the diagnosis of HCC. It has been hypothesised that an ageassociated decrease in DNA repair contributes to the development of HCC (Goukassian et al, 2000; Anisimov, 2007). Chronic hepatitis or cirrhosis raise AFP in $20 \%$ and $40 \%$ of patients, respectively, and tend to fluctuate in parallel with underlying inflammatory activity (Johnson, 2001; Kashyap et al, 2001). Also, ALT and AST ratio levels were significantly associated with the incidence of HCC (Dufour et al, 2000). Elevated AST activity is a surrogate marker for cirrhosis because of reduced plasma clearance of AST secondary to impaired function of sinusoidal cells (Park et al, 2000). Elevation of ALP enzyme can suggest intrahepatic or extrahepatic biliary obstruction, and occurs in hepatocellular injury to a lesser degree. Alkaline phosphatase has also been included in the Chinese University Prognostic Index (Yu et al, 2011). Albumin is an important factor for use in several scoring systems, such as the Child-Pugh and CLIP score systems (Ishizuka et al, 2012). The Alb levels at any time reflect its rate of synthesis but is not specific for liver disease and may occur in protein malnutrition, nephrotic syndrome, and chronic protein losing enteropathies (Rothschild et al, 1983). It is interesting to note that all of these blood markers are affected by liver inflammation and that none are precursors of liver fibrosis. In this study, the sensitivity and specificity of AFP for differentiating HCC from liver cirrhosis at a cutoff value of $400 \mathrm{Ul}^{-1}$ were $41 \%$ and $99 \%$, respectively. Hepatocellular carcinoma can produce a range of AFP values from normal to $>100000 \mathrm{Ul}^{-1}$ (Koteish and Thuluvath, 2002); AFP $>400-500 \mathrm{Ul}^{-1}$ is considered diagnostic for HCC, although fewer than half of patients may generate levels that are high (Bialecki and Di Bisceglie, 2005). With values of that magnitude, the specificity of AFP is close to $100 \%$ but at a cost to the sensitivity, which falls below 45\% (Gupta et al, 2003). This study used large number of patients to develop and validate a simple score predictive of HCC in chronic hepatitis C. In this study, after assessing a set of eight potential blood markers of HCC, we developed a predictive model consisting of age and four routine laboratory tests (AFP, AAR, ALP, and Alb). At the best cutoff, AUC, sensitivity, specificity, and accuracy for differentiating HCC from non-malignant liver cirrhosis were $0.99,97 \%, 96 \%$, and $96 \%$, respectively. Absolute specificity (100\%) was obtained to discriminate HCC from liver fibrosis (F1-F3) and F0. It is not easy to distinguish HCC from liver cirrhosis by imaging examination of the liver, procedures that to our knowledge can reach $50 \%$ efficiency (Patel et al, 2012). Hepatocellular carcinoma-AFP-routine test showed a diagnostic sensitivity for HCC of $97 \%$, higher than or comparable with that of other, sometimes invasive tools, including instrumental approaches. The diagnostic sensitivity of US was $84 \%$; CT for HCC was 79\%; and that of MRI was 77\% (Liovet et al, 1998). When combining the diagnostic ability of the different imaging modalities, the diagnostic sensitivity of US and MRI was $90 \%$, whereas addition of US to CT and MRI had a sensitivity of $82 \%$ and $88 \%$, respectively (Alaboudy et al, 2011). The diagnostic sensitivity of cytology by fine-needle aspiration biopsy for HCC may vary from 60 to $90 \%$, depending on the size of the lesion, on the examiner, and on the diameter of the puncturing needle (França et al, 2003). There is a need to develop non-invasive, sensitive blood marker to improve the early detection of HCC (Attallah et al, 2011; Kumada et al, 2011). The sensitivity and specificity for AFP were $61 \%$ and $71 \%$; for AFP-L3 were $37 \%$ and $92 \%$; and for DCP were $39 \%$ and $90 \%$, respectively. Values increased when AFP values were combined with AFP-L 3\% and DCP to $77 \%$ and 59\%, respectively (Sterling et al, 2009). Hepatocellular carcinoma-AFP-routine test score is much simpler and practical score than other scores such as the one developed by Castaldo et al (1995) and the other one developed by Ishida et al (2010). These scores showed lower sensitivity of $85 \%$ compared with our score of $97 \%$ and lower specificity of $74 \%$ vs $96 \%$. We used CLIP score (0-1) to define early-stage HCC. To stage patients in the CLIP score, multiple clinical indexes, such as Child-Pugh score, tumour morphology, AFP level, and presence of portal vein thrombosis are taken into account. The AUC for validated HCCART in patients with HCC who had only single tumour, absent vascular invasion, size $2 \mathrm{~cm}$ or less, and CLIP score (0-1) were 0.95 , $0.93,0.86,0.87$, respectively, compared with $0.72,0.71,0.71,0.50$, respectively, for AFP. The validation group confirmed the ability of 
Table 4. Diagnostic performances of validated HCC-ART score to discriminate 537 patients with HCC from patients with non-malignant chronic liver diseases $(n=1617)$

\begin{tabular}{|c|c|c|c|c|c|c|}
\hline Classification & Sen (\%) & Spe (\%) & PPV (\%) & NPV (\%) & $\mathrm{AC}(\%)$ & AUC \\
\hline $\begin{array}{l}\text { FO }(N=153) \text { vs } \mathrm{HCC} \\
\text { F1-F3 }(N=1073) \text { vs HCC } \\
\text { Liver cirrhosis child } \mathrm{A} \text { (no } 420 \text { ) vs HCC } \\
\text { Liver cirrhosis child } \mathrm{B} \text { (no } 25) \text { vs HCC Aged } 65 \pm 3.8 \text { years } \\
\text { Total liver cirrhosis (no }=445) \text { vs HCC }\end{array}$ & 90 & $\begin{array}{r}100 \\
100 \\
85 \\
52 \\
83\end{array}$ & $\begin{array}{r}100 \\
100 \\
89 \\
97 \\
87\end{array}$ & $\begin{array}{l}76 \\
97 \\
89 \\
20 \\
88\end{array}$ & $\begin{array}{l}98 \\
95 \\
88 \\
88 \\
87\end{array}$ & $\begin{array}{l}1.0 \\
1.0 \\
0.95 \\
0.86 \\
0.95\end{array}$ \\
\hline \multicolumn{7}{|l|}{ Number of nodules (no., \%) } \\
\hline $\begin{array}{l}\text { Single }(388,72.3 \%) \\
\text { Multiple }(149,27.7 \%)\end{array}$ & $\begin{array}{l}90 \\
92\end{array}$ & $\begin{array}{l}83 \\
83\end{array}$ & $\begin{array}{l}82 \\
65\end{array}$ & $\begin{array}{l}90 \\
97\end{array}$ & $\begin{array}{l}87 \\
85\end{array}$ & $\begin{array}{l}0.95 \\
0.95\end{array}$ \\
\hline \multicolumn{7}{|l|}{ Vascular invasion (no., \%) } \\
\hline $\begin{array}{l}\text { Absent }(528,98.3 \%) \\
\text { Present }(10,0.7 \%)\end{array}$ & $\begin{array}{r}87 \\
100\end{array}$ & $\begin{array}{l}83 \\
83\end{array}$ & $\begin{array}{l}83 \\
12\end{array}$ & $\begin{array}{r}88 \\
100\end{array}$ & $\begin{array}{l}85 \\
84\end{array}$ & $\begin{array}{l}0.93 \\
0.97\end{array}$ \\
\hline \multicolumn{7}{|l|}{ Size of nodules (no., \%) } \\
\hline $\begin{array}{l}<2(18,4 \%) \\
\geqslant 2(516,96 \%)\end{array}$ & $\begin{array}{l}69 \\
91\end{array}$ & $\begin{array}{l}81 \\
83\end{array}$ & $\begin{array}{l}10 \\
86\end{array}$ & $\begin{array}{l}99 \\
88\end{array}$ & $\begin{array}{l}81 \\
87\end{array}$ & $\begin{array}{l}0.86 \\
0.95\end{array}$ \\
\hline \multicolumn{7}{|l|}{ CLIP score } \\
\hline $\begin{array}{l}0-1(128,24 \%) \\
\geqslant 2(409,76 \%)\end{array}$ & $\begin{array}{l}73 \\
94\end{array}$ & $\begin{array}{l}83 \\
83\end{array}$ & $\begin{array}{l}81 \\
80\end{array}$ & $\begin{array}{l}56 \\
95\end{array}$ & $\begin{array}{l}81 \\
88\end{array}$ & $\begin{array}{l}0.87 \\
0.96\end{array}$ \\
\hline
\end{tabular}

Table 5. Diagnostic performances of validated AFP at $400 \mathrm{UI}^{-1}$ to discriminate 537 patients with HCC from patients with non-malignant chronic liver diseases $(n=1671)$

\begin{tabular}{|c|c|c|c|c|c|c|}
\hline Classification & Sen $\%$ & Spe \% & PPV\% & NPV\% & $\mathrm{AC} \%$ & AUC \\
\hline $\mathrm{FO}(\mathrm{N}=153)$ vs $\mathrm{HCC}$ & 54 & 100 & 100 & 30 & 62 & 0.70 \\
\hline F1-F3 $(N=1073)$ vs HCC & 40 & 100 & 100 & 83 & 84 & 0.70 \\
\hline Liver cirrhosis child A (no 420 ) vs HCC & 54 & 99 & 99 & 63 & 74 & 0.77 \\
\hline Liver cirrhosis child B (no 25) vs HCC Aged $65 \pm 3.8$ years & 59 & 100 & 100 & 30 & 64 & 0.73 \\
\hline Total liver cirrhosis $(\mathrm{no}=445)$ vs HCC & 54 & 99 & 98 & 64 & 74 & 0.77 \\
\hline \multicolumn{7}{|l|}{ Number of nodules (no., \%) } \\
\hline Single $(388 ; 72.3 \%)$ & 55 & 99 & 99 & 72 & 72 & 0.72 \\
\hline Multiple $(149,27.7 \%)$ & 52 & 99 & 95 & 86 & 77 & 0.69 \\
\hline \multicolumn{7}{|l|}{ Vascular invasion (no., \%) } \\
\hline Absent $(528,98.3 \%)$ & 46 & 99 & 98 & 61 & 70 & 0.71 \\
\hline Present $(10,0.7 \%)$ & 66 & 99 & 60 & 99 & 74 & 0.79 \\
\hline \multicolumn{7}{|l|}{ Size of nodules (no., \%) } \\
\hline$<2(18,4 \%)$ & 57 & 99 & 96 & 88 & 87 & 0.71 \\
\hline$\geqslant 2(516,96 \%)$ & 67 & 99 & 99 & 72 & 82 & 0.71 \\
\hline \multicolumn{7}{|l|}{ CLIP score } \\
\hline $0-1(128,24 \%)$ & 1 & 99 & 20 & 77 & 77 & 0.5 \\
\hline$\geqslant 2(409,76 \%)$ & 71 & 99 & 99 & 79 & 97 & 0.8 \\
\hline
\end{tabular}

HCC-ART score to diagnose HCC, especially those with earlystage disease. Receiver operating characteristic curves of simplified HCC-ART (without coefficient) showed AUC of 0.95 , sensitivity of $92 \%$, and specificity of $91 \%$ in the estimation group, and $0.91,83 \%$, and $86 \%$, respectively, in the validation group. Hepatocellular carcinoma-AFP-routine test score was developed and validated in Egyptian patients. Because the clinical characteristics included are common to clinical guidelines prepared by the American 
Table 6. Diagnostic performances of validated simplified HCC-ART score to discriminate 537 patients with HCC from patients with non-malignant chronic liver diseases $(n=1671)$

\begin{tabular}{|c|c|c|c|c|c|c|}
\hline Classification & Sen (\%) & Spe (\%) & PPV (\%) & NPV (\%) & $\mathrm{AC}(\%)$ & AUC \\
\hline $\begin{array}{l}\text { FO }(N=153) \text { vs HCC } \\
\text { F1-F3 }(N=1073) \text { vs HCC } \\
\text { Liver cirrhosis child } A \text { (no } 420 \text { ) vs HCC } \\
\text { Liver cirrhosis child } B(\text { no } 25) \text { vs HCC } \\
\text { Total liver cirrhosis (no }=445 \text { ) vs HCC Aged } 65 \pm 3.8 \text { years }\end{array}$ & 87 & $\begin{array}{r}100 \\
99 \\
84 \\
56 \\
83\end{array}$ & $\begin{array}{r}100 \\
99 \\
88 \\
98 \\
86 \\
\end{array}$ & $\begin{array}{l}60 \\
94 \\
84 \\
17 \\
84 \\
\end{array}$ & $\begin{array}{l}89 \\
95 \\
86 \\
86 \\
95 \\
\end{array}$ & $\begin{array}{l}0.99 \\
0.98 \\
0.91 \\
0.85 \\
0.91\end{array}$ \\
\hline \multicolumn{7}{|l|}{ Number of nodules (no., \%) } \\
\hline $\begin{array}{l}\text { Single }(388,72.3 \%) \\
\text { Multiple }(149,27.7 \%)\end{array}$ & $\begin{array}{l}87 \\
87\end{array}$ & $\begin{array}{l}83 \\
83\end{array}$ & $\begin{array}{l}81 \\
63\end{array}$ & $\begin{array}{l}88 \\
95\end{array}$ & $\begin{array}{l}85 \\
84\end{array}$ & $\begin{array}{l}0.91 \\
0.91\end{array}$ \\
\hline \multicolumn{7}{|l|}{ Vascular invasion (no., \%) } \\
\hline $\begin{array}{l}\text { Absent }(528,98.3 \%) \\
\text { Present }(10,0.7 \%)\end{array}$ & $\begin{array}{r}88 \\
100 \\
\end{array}$ & $\begin{array}{l}83 \\
83 \\
\end{array}$ & $\begin{array}{l}86 \\
11 \\
\end{array}$ & $\begin{array}{r}84 \\
100 \\
\end{array}$ & $\begin{array}{l}85 \\
83 \\
\end{array}$ & $\begin{array}{l}0.91 \\
0.97\end{array}$ \\
\hline \multicolumn{7}{|l|}{ Size of nodules (no., \%) } \\
\hline $\begin{array}{l}<2(18,4 \%) \\
\geqslant 2(516,96 \%)\end{array}$ & $\begin{array}{l}54 \\
89\end{array}$ & $\begin{array}{l}83 \\
83\end{array}$ & $\begin{array}{r}9 \\
81\end{array}$ & $\begin{array}{l}98 \\
90\end{array}$ & $\begin{array}{l}81 \\
85\end{array}$ & $\begin{array}{l}0.90 \\
0.91\end{array}$ \\
\hline \multicolumn{7}{|l|}{ CLIP score } \\
\hline $\begin{array}{l}0-1(128,24 \%) \\
\geqslant 2(409,76 \%)\end{array}$ & $\begin{array}{l}71 \\
92\end{array}$ & $\begin{array}{l}83 \\
83 \\
\end{array}$ & $\begin{array}{l}54 \\
83 \\
\end{array}$ & $\begin{array}{l}91 \\
92 \\
\end{array}$ & $\begin{array}{l}80 \\
87 \\
\end{array}$ & $\begin{array}{l}0.81 \\
0.94 \\
\end{array}$ \\
\hline
\end{tabular}

Association for the Study of Liver Diseases, this score might be applicable to non-Egyptian patients. The simplest score would be one that requires only variables that can be documented simply from routine laboratory tests without the need for sophisticated tests. Therefore, clinicians should consider using HCC-ART as diagnostic biomarkers for small HCC tumour size or as additional markers in HCC surveillance programme using ultrasonography. In addition, it is highly important to know whether this score will decrease in response to HCC therapy and reductions in tumour burden.

\section{CONFLICT OF INTEREST}

The authors declare no conflict of interest.

\section{REFERENCES}

Ahmad W, Ijaz B, Javed FT, Gull S, Kausar H, Sarwar MT, Asad S, Shahid I, Sumrin A, Khaliq S, Jahan S, Pervaiz A, Hassan S (2011) A comparison of four fibrosis indexes in chronic HCV: development of new fibrosiscirrhosis index (FCI). BMC Gastroenterol 11: 44.

Alaboudy A, Inoue T, Hatanaka K, Chung H, Hyodo T, Kumano S, Murakami T, Moustafa EF, Kudo M (2011) Usefulness of combination of imaging modalities in the diagnosis of hepatocellular carcinoma using Sonazoid ${ }^{\circledR}$ enhanced ultrasound, gadolinium diethylene-triamine-pentaacetic acid-enhanced magnetic resonance imaging, and contrast-enhanced computed tomography. Oncology 81(Suppl 1): 66-72.

Anisimov VN (2007) Biology of aging and cancer. Cancer Control 14: 23-31. Attallah AM, El-Far M, Abdel Malak CA, Zahran F, Farid K, Omran MM, Zagloul H, El-Deen MS (2011) Evaluation of cytokeratin-1 in the diagnosis of hepatocellular carcinoma. Clin Chim Acta 412: 2310-2315.

Barreiros AP, Piscaglia F, Dietrich CF (2012) Contrast enhanced ultrasound for the diagnosis of hepatocellular carcinoma (HCC): comments on AASLD guidelines. J Hepatol 57: 930-932.
Bialecki ES, Di Bisceglie AM (2005) Diagnosis of hepatocellular carcinoma. HPB (Oxford) 7: 26-34.

Bruix J, Sherman M (2005) Management of hepatocellular carcinoma. Hepatol 42: 1208-1236.

Bruix J, Sherman M, Llovet JM, Beaugrand M, Lencioni R, Burroughs AK, Christensen E, Pagliaro L, Colombo M, Rodés J. EASL Panel of Experts on HCC (2001) Clinical Management of Hepatocellular Carcinoma. Conclusions of the Barcelona-2000 EASL Conference. J Hepatol 35: 421-430.

Camma C, Cabibbo GP (2009) Prognostic scores for hepatocellular carcinoma: none is the winner. Liver Int 2009 29: 478-480.

Castaldo G, Oriani G, Cimino L, Topa M, Budillon G, Salvatore F, Sacchetti L (1995) Discriminant function based on serum analytes differentiates hepatocarcinoma from secondary liver neoplasia. Clin Chem 41: 439-443.

Choi BI, Lee JM (2010) Advancement in HCC imaging: diagnosis, staging and treatment efficacy assessments: imaging diagnosis and staging of hepatocellular carcinoma. J Hepatobiliary Pancreat Sci 17: 369-373.

Cross TJ, Rizzi P, Berry PA, B ruce M, Portmann B, Harrison PM (2009) King's score: an accurate marker of cirrhosis in chronic hepatitis C. Eur J Gastroenterol Hepatol 21: 730-738.

Dufour DR, Lott JA, Nolte FS, Gretch DR, Koff RS, Seeff LB (2000) Diagnosis and monitoring of hepatic injury. II. Recommendations for use of laboratory tests in screening, diagnosis, and monitoring. Clin Chem 46: 2050-2068.

França AV, Valério HM, Trevisan M, Escanhoela C, Sevá-Pereira T, Zucoloto S, Martinelli A, Soares EC (2003) Fine needle aspiration biopsy improves the diagnostic accuracy of cut needle biopsy of focal liver lesions. Acta Cytologica 47: 332-336.

Gonzalez SA, Keeffe EB (2011) Diagnosis of hepatocellular carcinoma: role of tumor markers and liver biopsy. Clin Liver Dis 15: 297-306.

Goukassian D, Gad F, Yaar M, Eller MS, Nehal US, Gilchrest BA (2000) Mechanisms and implications of the age-associated decrease in DNA repair capacity. FASEB J 14: 1325-1334.

Gupta S, Bent S, Kohlwes J (2003) Test characteristics of alphafetoprotein for detecting hepatocellular carcinoma in patients with hepatitis C. A systematic review and critical analysis. Ann Intern Med 139: 46-50.

Hsieh YY, Tung SY, Lee IL, Lee K, Shen CH, Wei KL, Chang TS, Chuang CS, Wu CS, Lin YH (2009) FibroQ: an easy and useful noninvasive test for 
predicting liver fibrosis in patients with chronic viral hepatitis. Chang Gung Med 32: 614-622.

Ishida H, Matsuo S, Inoue Y (2010) Evaluation of diagnostic performance of alpha-fetoprotein (AFP) and des-gamma-carboxy prothrombin (DCP) for HCV related hepatocellular carcinoma developed after long-term follow up. Rinsho Byor 58: 1065-1072.

Ishizuka M, Kubota K, Kita J, Shimoda M, Kato M, Sawada T (2012) Impact of an inflammation-based prognostic system on patients undergoing surgery for hepatocellular carcinoma: a retrospective study of 398 Japanese patients. Am J Surg 203: 101-106.

Johnson PJ (2001) The role of serum alpha-fetoprotein estimation in the diagnosis and management of hepatocellular carcinoma. Clin Liver Dis 5: $145-159$.

Kashyap R, Jain A, Nalesnik M, Carr B, Barnes J, Vargas HE, Rakela J, Fung J (2001) Clinical significance of elevated alpha-fetoprotein in adults and children. Dig Dis Sci 46: 1709-1713.

Kew MC (2012) Hepatocellular carcinoma in developing countries: Prevention, diagnosis and treatment. World J Hepatol 4: 99-104.

Koteish A, Thuluvath PJ (2002) Screening for hepatocellular carcinoma. J Vasc Interv Radiol 13: S185-S190.

Kumada T, Toyoda H, Kiriyama S, Tanikawa M, Hisanaga Y, Kanamori A, Tada T, Tanaka J, Yoshizawa H (2011) Predictive value of tumor markers for hepatocarcinogenesis in patients with hepatitis C virus. J Gastroenterol 46: 536-544.

Lai Q, Melandro F, Pinheiro RS, Donfrancesco A, Fadel BA, Levi Sandri GB, Rossi M, Berloco PB, Frattaroli FM (2012) Alpha-fetoprotein and novel tumor biomarkers as predictors of hepatocellular carcinoma recurrence after surgery: a brilliant star raises again. Int J Hepatol 2012: 893103.

Lehman EM, Wilson ML (2009) Epidemiology of hepatitis viruses among hepatocellular carcinoma cases and healthy people in Egypt: a systematic review and meta-analysis. Int J Cancer 124: 690-697.

Llovet JM, Bruix J, Fuster J, Castells A, Garcia-Valdecasas JC, Grande L, Franca A, Brú C, Navasa M, Ayuso MC, Solé M, Real MI, Vilana R, Rimola A, Visa J, Rodés J (1998) Liver transplantation for small hepatocellular carcinoma: the tumor-node-metastasis classification does not have prognostic power. Hepatol 27: 1572-1577.

Manichon AF, Bancel B, Durieux-Millon M, Ducerf C, Mabrut JY, Lepogam MA, Rode A (2012) Hepatocellular adenoma: evaluation with contrast-enhanced ultrasound and MRI and correlation with pathologic and phenotypic classification in 26 lesions. HPB Surg 2012: 418745 .
Murakami T, Mochizuki K, Nakamura H (2001) Imaging evaluation of the cirrhotic liver. Semin Liver Dis 21: 213-224.

Park GJ, Lin BP, Ngu MC, Jones DB, Katelaris PH (2000) Aspartate aminotransferases: alanine aminotransferases ratio in chronic hepatitis $\mathrm{C}$ infection: is it a predictor of cirrhosis? J Gastroenterol Hepatol 15: 386-390.

Patel M, Shariff MI, Ladep NG, Thillainayagam AV, Thomas HC, Khan SA, Taylor-Robinson SD (2012) Hepatocellular carcinoma: diagnostics and screening. Eval Clin Pract 18: 335-342.

Poynard T, Bedossa P, Opolon P (1997) Natural history of liver fibrosis progression in patients with chronic hepatitis C. The OBSVIRC, METAVIR, CLINIVIR, and DOSVIRC groups. Lancet 349: 825-832.

Pugh RN, Murray-Lyon IM, Dawson JL, Pietroni MC, Williams R (1973) Transection of the oesophagus for bleeding oesophageal varices. Br J Surg 60: 646-649.

Rothschild MA, Oratz M, Schreiber SS (1983) Effects of nutrition and alcohol on albumin synthesis. Alcohol Clin Exp Res 7: 28-30.

Sterling RK, Jeffers L, Gordon F, Venook AP, Reddy KR, Satomura S, Kanke F, Schwartz ME, Sherman M (2009) Utility of Lens culinaris agglutininreactive fraction of alpha-fetoprotein and des-gamma-carboxy prothrombin, alone or in combination, as biomarkers for hepatocellular carcinoma. Clin Gastroenterol Hepatol 7: 104-113.

Tandon P, Garcia-Tsao G (2009) Prognostic indicators in hepatocellular carcinoma: a systematic review of 72 studies. Liver Int 29: 502-510.

The Cancer of the Liver Italian Program (CLIP) Investigators (2000) Prospective validation of the CLIP score: a new prognostic system for patients with cirrhosis and hepatocellular carcinoma. Hepatology 31: 840-845.

Vallet-Pichard A, Mallet V, Nalpas B, Verkarre V, Nalpas A, Dhalluin-Venin V, Fontaine H, Pol S (2007) FIB-4: an inexpensive and accurate marker of fibrosis in $\mathrm{HC} \mathrm{V}$ infection. Comparison with liver biopsy and fibrotest. Hepatology 46: 32-36.

Yu MC, Chan KM, Lee CF, Lee YS, Eldeen FZ, Chou HS, Lee WC, Chen MF (2011) Alkaline phosphatase: does it have a role in predicting hepatocellular carcinoma recurrence? J Gastrointest Surg 15: 1440-1449.

This work is published under the standard license to publish agreement. After 12 months the work will become freely available and the license terms will switch to a Creative Commons AttributionNonCommercial-Share Alike 3.0 Unported License. 